\title{
Is there a consistent association between coronary heart disease and ischemic stroke caused by intracranial atherosclerosis?
}

\author{
Existe uma associação consistente entre doença coronária e acidente vascular cerebral \\ isquêmico causado por aterosclerose intracraniana?
}

Adriana B. Conforto', Claudia da Costa Leite², Cesar H. Nomura ${ }^{3}$, Edson Bor-Seng-Shu4 ${ }^{4}$ Raul D. Santos ${ }^{5}$

\begin{abstract}
Coronary heart disease and ischemic stroke are frequent coexistent conditions that share risk factors and pose major burdens to global health. Even though a clear relation has been established between extracranial internal carotid artery atherosclerosis and symptomatic or asymptomatic coronary heart disease, there is a gap in knowledge about the association between intracranial atherosclerosis and coronary heart disease. Intracranial atherosclerosis is associated with high risks of stroke recurrence and vascular death. More research and clinical trials are needed to answer whether early diagnosis of asymptomatic coronary heart disease and aggressive treatment can decrease the risk of vascular death in patients with ischemic stroke caused by intracranial atherosclerosis.
\end{abstract}

Key words: stroke, coronary disease, intracranial arteriosclerosis, atherosclerosis.

\section{RESUMO}

A doença coronária e o acidente vascular cerebral isquêmico são condições frequentemente associadas, que compartilham fatores de risco e representam grande sobrecarga à saúde mundial. Embora seja claramente reconhecida a relação entre a doença coronária sintomática ou assintomática e a aterosclerose da artéria carótida interna em sua porção extracraniana, há uma lacuna no conhecimento sobre a associação entre doença coronária e aterosclerose intracraniana, que por sua vez é associada a alto risco de recorrência de acidente vascular cerebral isquêmico e de morte por causas vasculares. É necessário avaliar se o diagnóstico precoce da doença coronária assintomática e seu tratamento agressivo podem diminuir o risco de morte por causas vasculares em pacientes com acidente vascular cerebral isquêmico ocasionado por aterosclerose intracraniana.

Palavras-Chave: acidente vascular cerebral, doença das coronárias, arteriosclerose intracraniana, aterosclerose.

Worldwide, 5.7 million deaths were attributed to stroke in $2004^{1}$. According to the Ministry of Health, stroke was the leading cause of mortality in Brazil in 2009, being responsible for 99,262 deaths². Coronary heart disease (CHD) also has a massive impact on Brazilian mortality and was responsible for 96,386 deaths within the same year.

Atherosclerosis is the main cause of CHD and a major cause of ischemic stroke (IS), the most common type of stroke $e^{3,4}$. Despite the systemic distribution of atherosclerosis, the incidence of CHD is high while the incidence of IS is relatively low in some countries, while the opposite can be found in other regions $^{5}$. CHD and IS share a number of risk factors and some, but not all etiopathogenic features. Risk factors for both conditions are similar, but not exactly the same, and may play different roles in overall risk. For example, the population risk attributed to arterial hypertension for all strokes was estimated to be $90.3 \%$ in the INTERSTROKE study ${ }^{6}$, while for CHD, the attributed risk was $17.9 \%$ in INTERHEART ${ }^{7}$. These discrepancies partially reflect the heterogeneous etiopathogenic mechanisms of different types of IS and hemorrhagic strokes,

\footnotetext{
${ }^{1}$ Professor of Neurology, Stroke group, Neurology Clinical Division, Hospital das Clínicas/São Paulo University and Center for Interdisciplinary Research on Applied Neurosciences (NAPNA), University of São Paulo Researcher, Instituto Israelita de Ensino e Pesquisa, Hospital Israelita Albert Einstein; São Paulo SP, Brazil;

${ }^{2}$ Professor of Radiology, Radiology Department, Hospital das Clínicas, São Paulo University, São Paulo SP, Brazil;

${ }^{3} \mathrm{MD}, \mathrm{PhD}$, Radiology Department and Heart Institute, Hospital das Clínicas, São Paulo University, São Paulo SP, Brazil;

${ }^{4}$ Professor of Neurosurgery, Neurosurgery Division, Hospital das Clínicas, São Paulo University, São Paulo SP, Brazil;

${ }_{5}^{5}$ Professor of Cardiology, Lipid Clinic, Heart Institute, Hospital das Clínicas, São Paulo University, São Paulo SP, Brazil;

Correspondence: Adriana B. Conforto; Divisão de Clínica Neurológica, HC/FMUSP; Avenida Dr. Enéas C. Aguiar 255 / $5084 ; 05409-010$ São Paulo SP - Brasil;

E-mail:adriana.conforto@gmail.com

Conflict of interest: There is no conflict of interest to declare.
}

Received 12 September 2012; Received in final form 09 October 2012; Accepted 17 October 2012. 
compared to CHD. A better understanding of mechanisms underlying IS and CHD is crucial to develop effective preventive and treatment strategies.

While myocardial infarction is caused by coronary atherosclerosis in almost $100 \%$ of cases, IS can have various etiologies: large-artery atherosclerosis, cardiac embolism, small-vessel disease, or less common causes such as arterial dissection and hematological disorders, among others. IS etiology may remain undetermined in up to 30-40\% of the cases despite extensive investigation, particularly in young adults ${ }^{8-10}$.

Atherosclerosis explains $20-40 \%$ of IS cases in different populations, depending on genetic and demographic characteristics, as well as on prevalence of risk factors for vascular disease and extension of investigation of stroke etiology ${ }^{8,11}$. In patients with IS due to atherosclerosis, arterial occlusion or the source of artery-to-artery embolism may be found in extracranial or intracranial arteries.

In the past years, attention has been given to the importance of intracranial atherosclerosis as a cause of IS. Traditionally, it is considered that intracranial atherosclerosis is more common in Black, Hispanic and Asian individuals. It has been estimated that intracranial atherosclerosis is responsible for 5-10\% of IS in Whites, and 33\% in Blacks, Asians or Hispanics respectively ${ }^{12-15}$. Intracranial atherosclerosis may be the first cause of IS around the world, due to its high prevalence in China and other highly populated countries in Asia. It has been hypothesized that, during human diversification, genotypes of individuals that remained in Europe enhanced resistance to intracranial atherogenesis. In Asian countries, a healthy lifestyle may have had a protective effect against intracranial atherosclerosis in the past. However, protection was dramatically weakened by cultural changes that drove nutrition and physical activity habits closer to those of Western countries ${ }^{16}$.

No epidemiological studies about the role of intracranial atherosclerosis in IS in Brazil have been published, but characteristics of IS survivors with basilar artery occlusive disease have been described in a small hospital-based regis$\operatorname{try}^{17}$. In this series, atherosclerosis was the etiology of IS in $87.5 \%$ of the cases. Most patients (90\%) had more than one traditional risk factor for vascular disease, which is consistent with other series of intracranial atherosclerosis and IS or transient ischemic attack (TIA) worldwide. Typically, patients with IS and severe intracranial atherosclerosis present metabolic syndrome, dyslipidemia and type 2 diabetes mellitus more often than those with moderate intracranial atherosclerosis ${ }^{13}$. It is thus expected that these patients be more prone to aggressive systemic atherosclerosis. Indeed, in Chinese patients with IS and coexistent coronary, intracranial and extracranial atherosclerosis followed on average by 65 months, the rate of recurrent vascular events or death was $89 \%^{18}$.
In the WASID study (Warfarin-Aspirin Symptomatic Intracranial Disease Trial), where 569 patients with IS caused by intracranial atherosclerosis were randomized to either aspirin or warfarin in the United States, the yearly risk of death from vascular causes was $1.8 \%$ in the aspirin group and $3.1 \%$ in the warfarin group ${ }^{19}$. After a median follow-up of 1.8 years, myocardial infarctions occurred in $2.5 \%$ of the patients in the aspirin group, and in $4.2 \%$ of those in the warfarin group. Stroke or death occurred in $23.6 \%$ of the patients in the aspirin group, and in $24.6 \%$ of those in the warfarin group.

In comparison with the WASID study, the SAMMPRIS (Stenting and Aggressive Medical Management for Preventing Recurrent Stroke in Intracranial Stenosis study) trial employed a more aggressive medical treatment that included aspirin, addition of clopidogrel for 90 days, hypertension treatment (target: systolic blood pressure < $140 \mathrm{mmHg}$; <130 $\mathrm{mmHg}$ in diabetics), statins (target: LDL-cholesterol $<70 \mathrm{mg} / \mathrm{dL}$ ) and a lifestyle modification program ${ }^{20}$. In addition patients were randomized to either medical treatment alone or medical treatment plus intracranial angioplasty and stenting. In SAMMPRIS, the probability of stroke or death in patients submitted to medical treatment alone $(12.2 \%)$ was less than that described in WASID at 1 year, however the myocardial infarction incidence was still 4\%. Together, these results underscore the need to further understand the link between intracranial atherosclerosis and CHD.

\section{CORONARY HEART DISEASE AND ISCHEMIC STROKE}

Within the first year after stroke, recurrence of a cerebrovascular event is responsible for $20-27 \%$ of hospital admissions however, over ten years, the risk of recurrence decreases, while the risk of other vascular events increases ${ }^{21,22}$. Within the first year after stroke or TIA of various causes, the risk of death or hospital admission due to vascular causes has been estimated as $10 \%$ and $20 \%$ respectively in patients without and with $\mathrm{CHD}^{23-24}$. In the Northern Manhattan Study (NOMAS), the risk of nonfatal or fatal stroke was $18.3 \%$ five years after stroke, and the risk of cardiac events was $8.6 \%$. Also after a median follow-up of 4.9 years, mortality due to stroke was $6.4 \%$ and cardiac mortality, $3.7 \%^{25}$.

Within the first year after IS specifically caused by atherosclerosis, the risk of death from myocardial infarction has been estimated to be $1.9 \%{ }^{26}$. In The Stroke Prevention by Aggressive Reduction in Cholesterol trial (SPARCL), at a median follow-up of 4.9 years after non-disabling, non-cardioembolic IS or hemorrhagic stroke or TIA, 5.1\% of the patients that did not receive statins had a major coronary event ${ }^{27}$. While recurrent stroke rates decreased during follow-up, the rates of major coronary events remained stable. For this reason, it has been proposed that stroke and TIA, particularly 
when caused by atherosclerosis, should be considered as coronary risk equivalents ${ }^{28}$. A statement in this regard from the American Heart Association/American Stroke Association has recently been published ${ }^{29}$.

Involvement of the coronary arteries is common in patients with non-cardioembolic IS or TIA. In the Reduction of Atherosclerosis for Continued Health (REACH) study, established CHD was present in $37.9 \%$ of the patients with IS or TIA ${ }^{23}$. However, this represents only the tip of the iceberg, if asymptomatic CHD is also considered. Over the past years, accumulated evidence showed that the association between asymptomatic IS and CHD is frequent and may be clinically relevant.

In the Multiple Atherosclerosis Site in Stroke study (MASS), 267 autopsies were performed after fatal strokes. Coronary plaques were observed in $69.7 \%$ of patients with IS and no history of $\mathrm{CHD}^{30}$. When subgroups were analyzed, coronary plaques were present in $77.1 \%$ of the cases with atherothrombotic, $65.5 \%$ of the cases with cardioembolic, $79 \%$ of cases with lacunar and $63 \%$ of undetermined cause IS. These high rates of coronary plaques in IS subjects without history of CHD symptoms contrasted with the $24.7 \%$ prevalence of coronary atherosclerosis in 442 patients with other neurologic diseases.

In the PRECORIS (Prevalence of asymptomatic Coronary artery disease in Ischemic Stroke study), computed coronary angiography showed stenosis $\geq 50 \%$ in $20 \%$ of 274 patients with non-disabling, non-cardioembolic IS or TIA ${ }^{31}$. By performing invasive coronary angiography in 315 patients with IS of various etiologies and no CHD symptoms, researchers of the AMISTAD (Asymptomatic Myocardial Ischemia in Stroke and Atherosclerotic Disease) study revealed coronary plaques in $62 \%$, and coronary stenosis $\geq 50 \%$ in $26 \%$ of the cases ${ }^{32}$.

\section{RECOMMENDATIONS FOR EVALUATION OF CORONARY HEART DISEASE IN PATIENTS WITH ISCHEMIC STROKE OR TRANSIENT ISCHEMIC ATTACK}

Despite the evidence of association between $\mathrm{CHD}$ and IS or TIA, there are no strong recommendations for CHD screening in asymptomatic patients with cerebrovascular disorders ${ }^{24}$. The bulk of the work in the field has focused on the strong association between extracranial internal carotid artery atherosclerosis and $\mathrm{CHD}^{33}$. About $25-60 \%$ of patients with extracranial carotid disease and no history of CHD show abnormal results in noninvasive or invasive cardiac testing. CHD risk is 11 times higher in patients with extracranial internal carotid artery atherosclerosis than in the general population. Death from vascular causes, myocardial infarction (MI) or stroke occurs in $6 \%$ of patients with stroke caused by extracranial internal carotid artery atherosclerosis, within the first year post-stroke. Considering this strong relation between both conditions, the American Heart Association/ American Stroke Association published recommendations for CHD investigation in IS or TIA patients in $2003^{33}$, as outlined in the Table.

According to these guidelines, noninvasive testing for CHD is indicated in patients with stroke or TIA caused by extracranial internal carotid artery atherosclerosis, in high-risk patients according to Framingham CHD risk stratification, and possibly in those with stroke subtypes clearly related to atherosclerosis in intracranial arteries or extracranial vertebral arteries.

However, whether asymptomatic CHD or elevated CHD risk are significantly more common in patients with IS caused by atherosclerosis than by other etiologies, or in patients with IS of undetermined cause, are still controversial issues. Furthermore, both the use of the Framingham risk stratification equation and the diagnosis of IS due to intracranial atherosclerosis are faced with challenges.

Initially, the Framingham stratification equation incorporated age, sex, LDL-cholesterol, HDL-cholesterol, blood pressure, diabetes mellitus and smoking status to estimate CHD risk (MI, death caused by MI and angina) in 10 years ${ }^{34}$. Scores were shown to have good predictive values for CHD events in the United States. In 2002, the stratification system was modified, for screening and treatment of patients with dyslipidemia $^{35}$. Among the changes, the "diabetes mellitus" item was eliminated from the algorithm because this disease was considered as a coronary equivalent. CHD risk was classified as low $(<10 \%)$, intermediate $(10-20 \%)$ or high $(>20 \%)^{35,36}$. A caveat of using the Framingham risk stratification in patients with IS or TIA is that the system was created for evaluation of the general population and not to individual patients with

Table. Summary of recommendations for evaluation of coronary heart disease in ischemic stroke or transient ischemic attack ${ }^{33}$.

\begin{tabular}{ll} 
Diagnosis & Recommendation \\
\hline & $\begin{array}{l}\text { All patients should be assessed for cardiovascular risk, preferably based on existing recommendations to } \\
\text { identify those at greater likelihood of morbidity and mortality from asymptomatic CHD. } \\
\text { Risk factor reduction is recommended in all cases, regardless of the decision to perform noninvasive } \\
\text { cardiac testing. } \\
\text { Ischemic stroke }\end{array} \quad \begin{array}{ll}\text { Noninvasive testing for CHD should be considered in patients with high CHD risk factor scores based on } \\
\text { Framingham algorithms (10-year CHD risk } \geq 20 \%) .\end{array}$ \\
& If there are few risk factors for CHD, no carotid artery disease, or stroke etiology is not clearly related to \\
& atherosclerosis, routine screening is not recommended. \\
\hline \multirow{2}{*}{ Carotid artery disease } & Selected patients with high cardiovascular risk profiles and symptoms of brain ischemia in the presence \\
& of significant carotid disease should be considered for noninvasive testing for coronary heart disease.
\end{tabular}

CHD: coronary heart disease. 
cardiovascular disease. In the Framingham cohort, the number of risk factors associated with greater likelihood of CHD was lower in patients with history of CHD or stroke, than in patients without cardiovascular disease ${ }^{37}$.

Also, stroke etiologies are heterogeneous. Patients with IS caused by extracranial internal carotid artery atherosclerosis are known to have greater CHD risk than the general population, but CHD risks have been less investigated in IS caused by other etiologies, such as intracranial atherosclerosis or non-atherosclerotic causes. The IV Brazilian guidelines of Atherosclerosis Prevention ${ }^{38}$ include any stroke or TIA as a coronary equivalent, leading to assignment of a Framingham score $>20 \%$. Considering that not all IS or TIA are caused by atherosclerosis, this strategy is open to discussion $^{29}$. The absence of significant differences in burden of asymptomatic CHD in autopsies of patients with different IS etiologies may indicate that indeed, patients with any IS are at increased risk, or may reflect low power due to small sample sizes in IS subgroups ${ }^{30}$.

For those who do not support the concept that a Framingham score of at least $20 \%$ should be attributed to a patient with ischemic stroke not caused by carotid artery disease, or in whom intracranial atherosclerosis was not diagnosed (negative or incomplete investigation), the choice to use the score for stratification of vascular risk can be misleading. In theory, it is possible that cut-off values for estimation of CHD risk may be lower in patients with IS compared to the general population, or in miscigenated populations compared to North-American and European populations. The Framingham stratification system was validated in countries of predominantly European ancestry, and its predictive value in miscigenated populations remains to be determined. Therefore, by limiting cardiac testing to patients with IS and Framingham scores $>20 \%$ in 10 years, physicians might lose the opportunity to diagnose CHD in asymptomatic patients at moderate or high risk of MI death. The inclusion of additional criteria or biomarkers such as family history of early CHD, chronic renal insufficiency or calcium scores, among others, may refine stratification but this approach has not been thoroughly tested in patients with $\mathrm{IS}^{38}$.

Another defying task for physicians that treat patients with stroke is to properly investigate and diagnose atherosclerosis as a cause of IS or TIA, in the absence of extracranial internal carotid artery atherosclerosis. Appropriate interpretation of clinical, laboratory and imaging findings is crucial to diagnose IS caused by atherosclerosis in the extracranial vertebral artery, or in intracranial arteries. Tests such as intracranial magnetic resonance or computed tomography angiography and transcranial Doppler are key for diagnosis of intracranial artery disease ${ }^{39}$. If physicians do not acknowledge intracranial artery disease as a cause of IS that deserves investigation, such tests may never be ordered, leading to underdiagnosis of this condition. For instance, diagnosis of basilar artery occlusive disease may be missed if investigation of intracranial arteries is not performed in a patient with TIAs presenting with pure motor hemiparesis and mild or absent extracranial internal carotid artery atherosclerosis ${ }^{40}$. If the Framingham score in such a patient points to a $10 \%$ CHD risk in 10 years, noninvasive cardiac investigation is unlikely to be performed, and aggressive medical treatment for control of vascular risk factors is less likely to be undertaken.

Considering that patients with intracranial artery occlusive disease are considered to be at high risk of recurrence and death from cardiovascular events, and that aggressive treatment of atherosclerosis has been suggested to be key in these cases, underdiagnosis of this condition may have serious consequences for overall vascular risk. The fact that the relation between extracranial internal carotid artery atherosclerosis and CHD has been well recognized should not cloud the need to determine if there is a similar association between atherosclerosis in other cervicocranial arteries, including intracranial arteries, and CHD risk. Few studies have addressed this issue over the past years.

\section{CORONARY AND INTRACRANIAL ATHEROSCLEROSIS}

The composition of the arterial wall and the magnitude of hemodynamic stress sustained by coronary and large intracranial arteries are different. In autopsies from Japanese individuals, the amounts of esterified to total cholesterol, and of chondroitin sulfate-46 in total glycosaminoglycans were lower, while heparin sulfate content was higher in normal intima of intracranial arteries than in coronary arteries and the aor$\mathrm{ta}^{41}$. In autopsies, lipids have been reported to be predominant components of coronary plaques, while fibrotic content seems to predominate in intracranial plaques. Plaques rich in lipids are more likely to ulcerate, triggering acute thrombosis, than fibrous plaques ${ }^{5}$. Also, it has been suggested that atherosclerosis may often affect either extra- or intracranial arteries, instead of involving both arterial beds. It has been proposed that pathogenesis of intracranial and extracranial atherosclerosis may differ, for example concerning metabolic abnormalities and inflammation ${ }^{42}$.

Correlations between coronary and internal carotid artery plaques have been widely described, but few studies have explored the coexistence of coronary and intracranial atherosclerosis. History of CHD has been reported to be more frequent in patients with extracranial carotid artery atherosclerosis than in those with middle cerebral artery stenosis but overall, insufficient information is available about intracranial atherosclerosis ${ }^{42}$.

In Japan, autopsies performed in the 1960s revealed a lower degree of atherosclerosis in the coronary arteries, and a higher burden in the circle of Willis, with respect to 
Americans. On the other hand, in India intracranial and coronary atherosclerosis were found to be significantly correlated $^{43}$. Consistent with other reports, coronary atherosclerosis was more frequent than intracranial atherosclerosis in younger individuals, and the correlation between the progression of atherosclerosis with age was more marked for coronary than for cerebral atherosclerosis. While coronary atherosclerotic burden increased gradually until age 40 and then evolved sharply with age, intracranial atherosclerosis was more evident after age 40 and continued to build gradually until the eight decade. Moreover, calcified and complicated plaques were common in coronary but not in intracranial plaques after age 40.

Characteristics and distribution of plaques in coronary and intracranial arterial beds may differ according to ethnicity and exposure to risk factors. In contrast with the results reported in India, fatty streaks and fibrous plaques were present in intracranial arteries already in the second decade of life, in autopsies performed in New Orleans in the 1960 s and early $1970 \mathrm{~s}^{43,44}$.

In regard to coronary atherosclerosis, ethnic and regional differences are also well established. For instance, in a study that included more than 17,000 asymptomatic Brazilian, Portuguese and American subjects, Santos et al. demonstrated different prevalence values and severity levels of coronary artery calcification, a surrogate marker of CHD. Dissimilarities remained significant even after adjustments were made for traditional risk factors for atherosclerosis ${ }^{45}$.

Genetic factors have been pointed out as candidate reasons to explain the "Hispanic paradox", i.e. the relatively lower risk of CHD death in Hispanics compared to non-Hispanics in the USA despite high prevalence of risk factors for vascular disease and lower socioeconomic status ${ }^{46}$. It is possible that genetic and environmental factors modulate the involvement of coronary and cervicocephalic arteries by different mechanisms and at different degrees in various populations. Therefore, the coexistence, severity and progress of atherosclerotic lesions in diverse arterial beds may be more frequent in some populations than in others, with likely implications on causes of vascular death. Until now, this remains an open question.

\section{ASYMPTOMATIC CORONARY AND INTRACRANIAL ATHEROSCLEROSIS IN PATIENTS WITH ISCHEMIC STROKE}

Relatively few studies have investigated the relation between asymptomatic coronary and intracranial atherosclerosis, or identified biomarkers/imaging tests that might help stratify patients with intracranial atherosclerosis for noninvasive investigation of CHD. More than $50 \%$ of 168 patients with IS or TIA and intracranial atherosclerosis without overt
CHD symptoms were reported to have perfusion defects on stress-rest $\mathrm{SPECT}^{47}$. In these patients, intracranial internal carotid artery stenosis, symptomatic vertebrobasilar stenosis and presence of high levels of lipoprotein and homocysteine were independently associated with silent myocardial ischemia. In another study, lipoprotein-associated phospliphase A(2), a novel cardiovascular risk biomarker, was found to be the only predictor of new vascular events in patients with IS or TIA and intracranial atherosclerosis ${ }^{48}$.

In Korea, 71 patients with IS of various etiologies and no history of CHD underwent computed tomography coronary angiography ${ }^{49}$. Coronary stenosis $>50 \%$ was identified in $25.4 \%$ of the cases. Patients with coronary stenosis had greater rates of IS caused by atherosclerosis (38.9\%), intracranial arterial stenosis $(61.1 \%)$ and extracranial carotid stenosis $(66.7 \%)$ than patients without coronary stenosis. Older age and extracranial internal carotid artery atherosclerosis, but not intracranial atherosclerosis, were independent predictors of CHD. However, statistical power for detection of a significant association between CHD and IS caused by intracranial atherosclerosis was low, since only 14 patients were diagnosed with IS caused by atherosclerosis (extra- or intracranial). Contraindications for computed tomography coronary angiography (renal failure, inability to cooperate with the test) or for extracranial and intracranial magnetic resonance angiography limited sample size.

In contrast with the conclusions from this study, another Korean study analyzed coronary computed tomography angiographies from 1,304 patients with IS and reported coronary artery stenosis $\geq 50 \%$ in $32.2 \%$ of the patients ${ }^{50}$. Significant correlations were found between coronary atherosclerosis and stenosis of the extracranial carotid, intracranial vertebral and basilar arteries, but not with anterior, middle and posterior cerebral arteries. These results suggested that the relation between coronary and intracranial atherosclerosis may be present for some, but not all intracranial arteries. Therefore, studying "intracranial atherosclerosis" instead of disease in distinct intracranial arteries may bias results, obscuring the relation between intracranial and coronary lesions.

In addition, a Japanese study that evaluated 104 patients with IS patients with no symptoms of CHD reported intracranial atherosclerosis and the metabolic syndrome as the strongest predictors of CHD, diagnosed by coronary computed tomography or coronary angiography ${ }^{51}$.

Differences in methodology, sample size, genetic as well as environmental factors may explain the discrepancies found in literature.

\section{CONCLUSIONS}

CHD and IS are frequent coexistent conditions that share risk factors and pose major burdens to global health. 
Intracranial atherosclerosis, the most frequent cause of IS in Asia and possibly the most likely cause worldwide, is associated with high risks for stroke recurrence and vascular death. Even though a clear relation has been established between extracranial internal carotid artery atherosclerosis and symptomatic or asymptomatic CHD, there is a gap in knowledge about the association between intracranial atherosclerosis, and CHD. Intracranial atherosclerosis is associated with high risk for stroke recurrence and vascular death. There is a need for more research in this field, as well as for clinical trials that answer whether early diagnosis of asymptomatic CHD and aggressive treatment, whether or not be restricted to medical treatment, can decrease the risk of vascular death in patients with IS caused by intracranial atherosclerosis.

\section{References}

1. WHO. Burden of Disease Statistics. Geneva, Switzerland: World Health Organization. http://www.who.int/healthinfo/global_burden_disease/ GBD_report_2004update_part2.pdf. Last accessed September 5 $5^{\text {th }}, 2012$.

2. Indicadores e Dados Básicos - Brasil - 2009. Disponível em: http:// tabnet.datasus.gov.br/cgi/idb2009.

3. Feigin VL, Lawes CCM, Bennett DA, Barker-Collo SL, Parag V. Worldwide stroke incidence and early case fatality reported in 56 populationbased studies: a systematic review. Lancet Neurol 2009;8:355-369.

4. Cabral NL, Gonçalves ARR, Longo AL, et al. Incidence of stroke subtypes, prognosis and prevalence of risk factors in Joinville, Brazil: a 2-year community based study. J Neurol Neurosurg Psychiatry 2009;80:755-761.

5. Puddu P, Puddu GM, Bastagli L, Massarelli G, Muscari A. Coronary and cerebrovascular atherosclerosis: two aspects of the same disease or two different pathologies? Arch Gerontol Geriatr 1995;20:15-22.

6. O'Donnell MJ, Xavier D, Liu L, et al. Risk factors for ischaemic and intracerebral haemorrhagic stroke in 22 countries (the interstroke study): a case-control study. Lancet 2010;376:112-123.

7. Yusuf S, Hawken S, Ôunpuu S, et al. Effect of potentially modifiable risk factors associated with myocardial infarction in 52 countries (the interheart study): case-control study. Lancet 2004;364:937-952.

8. Albers GW, Amarenco P, Easton JD, Sacco RL, Teal P. Antithrombotic and thrombolytic therapy for ischemic stroke. Chest 2001;119:300S-320S.

9. Ay $\mathrm{H}$. Advances in the diagnosis of etiologic subtypes of ischemic stroke. Curr Neurol Neurosci Rep 2010;10:14-20.

10. Yamamoto FI. Ischemic stroke in young adults: an overview of etiological aspects. Arq Neuropsiquiatr 2012;70:462-466.

11. Grau AJ, Weimar C, Buggle F, et al. Risk factors, outcome, and treatment in subtypes of ischemic stroke: the German Stroke Data Bank. Stroke 2001;32:2559-2566

12. Gorelick PB, Wong KS, Bae HJ, Pandey DK. Large artery intracranial occlusive disease: a large worldwide burden but a relatively neglected frontier. Stroke 2008;39:2396-2399.

13. Arenillas JF. Intracranial atherosclerosis: current concepts. Stroke 2011;42(Suppl):S20-S23.

14. Sacco RL, Kargman DE, Gu Q, Zamanillo MC. Race-ethnicity and determinants of intracranial atherosclerotic cerebral infarction: the Northern Manhattan Stroke Study. Stroke 1995;26:14-22.

15. Mazighi M, Labreuche J, Gongora-Rivera F, Duyckaerts C, Hauw JJ, Amarenco P. Autopsy prevalence of intracranial atherosclerosis in patients with fatal stroke. Stroke 2008;39:1142-1147.

16. Mak W, Cheng TS, Chan KH, Cheung RTF, Ho SL. A possible explanation for the racial difference in distribution of large-arterial cerebrovascular disease; ancestral European settlers evolved genetic resistance to atherosclerosis, but confined to the intracranial arteries. Med Hypotheses 2005;65:637-648.

17. Ciriaco JGM, Leite CC, Martin MGM, et al. Basilar artery occlusive disease in stroke survivors in a multiethnic population. Clin Neurol Neurosurg 2009;112:233-236.
18. Man BL, Fu YP, Chan YY, et al. Long-term outcomes of ischemic stroke patients with concurrent intracranial and extracranial stenoses and ischemic heart disease. Cerebrovasc Dis 2010;29:236-241.

19. Chimowitz MI, Lynn MJ, Howlett-Smith H, et al. Comparison of warfarin and aspirin for symptomatic intracranial stenosis. N Engl J Med 2005;352:1305-1316.

20. Chimowitz MI, Lynn MJ, Derdeyn CP, et al. Stenting versus aggressive medical therapy for intracranial arterial stenosis. $N$ Engl $J$ Med 2011;365:993-1003.

21. Hardie K, Hankey GJ, Jamrozik K, Broadhurst R, Anderson C. Ten-year survival after first-ever stroke in the Perth Community Stroke Study. Stroke 2003;34;1842-1846.

22. Anderson CS, Jamrozik KD, Broadhurst RJ, Stewart-Wynne EG. Predicting survival for 1 year among different subtypes of stroke: results from the Perth Community Stroke Study. Stroke 1994;25:1935-1944

23. Steg PG, Bhatt DL, Wilson PW, et al. One-year cardiovascular event rates in outpatients with atherothrombosis. JAMA 2007; 297:1197-1206.

24. Sander D, Carolei A, Diehm C, Hennerici MG. Challenges to the management of high-risk stroke patients with multiple-site occlusive vascular disease. Cerebrovasc Dis 2011;31:315-321.

25. Dhamoon MS, Sciacca RR, Rundek T, Sacco RL, Elkind MS. Recurrent stroke and cardiac risks after first ischemic stroke: the Northern Manhattan Study. Neurology 2006; 14:641-646.

26. Touzé E, Varenne O, Chatellier G, et al. Risk of myocardial infarction and vascular death after transient ischemic attack and ischemic stroke: a systematic review and meta-analysis. Stroke 2005;36:2748-2755.

27. Amarenco P, Goldstein LB, Sillesen H, et al. Coronary heart disease risk in patients with stroke or transient ischemic attack and no known coronary heart disease: findings from the Stroke Prevention by Aggressive Reduction in Cholesterol Levels (SPARCL) Trial. Stroke 2010;41:426-430.

28. Fitchett DH, Goodman SG, Langer A. Ischemic stroke: a cardiovascular risk equivalent? Lessons learned from the Stroke Prevention by Aggressive Reduction in Cholesterol Levels (SPARCL) trial. Can J Cardiol 2008;24:705-708.

29. Lackland DT, Elkind MS, D’Agostino R Sr, et al. Inclusion of stroke in cardiovascular risk prediction instruments: a statement for healthcare professionals from the American Heart Association/American Stroke Association. Stroke 2012;43:1998-2027.

30. Gongora-Rivera F, Labreuche J, Jaramillo A, Steg PA, Hauw JJ, Amarenco P. Autopsy prevalence of coronary atherosclerosis in patients with fatal stroke. Stroke 2007;38:1203-1210.

31. Calvet D, Touzé E, Varenne O, Sablayrolles JL, Weber S, Mas JL. Prevalence of asymptomatic coronary artery disease in ischemic stroke.patients: the PRECORIS study. Stroke 2010;121:1623-1629.

32. Amarenco P, Lavallée PC, Labereuche L, et al. Prevalence of coronary atherosclerosis in patients with cerebral infarction. Stroke 2011;42:22-29. 
33. Adams RJ, Chimowitz MI, Alpers JS, et al. Coronary risk evaluation in patients with transient ischemic attack and ischemic stroke: a scientific statement for healthcare professionals from the stroke council and the council on clinical cardiology of the American Heart Association/American Stroke Association. Stroke 2003;34:2310-2322.

34. D’Agostino RB, Sr, Grundy, S, Sullivan, LM, Wilson, P, CHD Risk Prediction Group. Validation of the Framingham coronary heart disease prediction scores: results of a multiple ethnic groups investigation. JAMA 2001;286:180-187.

35. National Cholesterol Education Program (NCEP) Expert Panel on Detection, Evaluation, and Treatment of High Blood Cholesterol in Adults (Adult Treatment Panel, III). Third Report of the National Cholesterol Education Program (NCEP) Expert Panel on Detection, Evaluation, and Treatment of High Blood Cholesterol in Adults (Adult Treatment Panel III) final report. Circulation 2002;106:3143-3421.

36. Grundy SM, Pasternak R, Greenland P, et al. Assessment of cardiovascular risk by use of multiple-risk-factor assessment equations: a statement for healthcare professionals from the American Heart Association and the American College of Cardiology. Circulation 1999;100:1481-1492.

37. D'Agostino RB, Russell MW, Huse DM, et al. Primary and subsequent coronary risk appraisal: new results from the Framingham Study. Am Heart J 2000;139:272-281.

38. Sposito AC, Caramelli B, Fonseca FA. IV Brazilian Guideline for Dyslipidemia and Atherosclerosis prevention: Department of Atherosclerosis of Brazilian Society of Cardiology. Arq Bras Cardiol 2007;88(Suppl 1):S2-S19.

39. Feldmann E. Stroke Outcomes and Neuroimaging of Intracranial Atherosclerosis (SONIA): Design of a prospective, multicenter trial of diagnostic tests. Neuroepidemiology 2004;23:23-32.

40. Conforto AB, Ciríaco JGM, Yamamoto FI, et al. Avoiding pitfalls in diagnosing basilar artery occlusive disease: clinical and imaging clues — case report. Sao Paulo Med J 2010;128:171-173.

41. Nakamura M, Imaizumi K, Shigemi U, Nakashima Y, Kikuchi Y. Cerebral atherosclerosis in Japanese. Part 5: relationship between cholesterol deposition and glycosaminoglycans. Stroke 1976;7:594-598.
42. Bang OY, Lee PH, Yoon SR, Lee MA, Joo IS, Huh K. Inflammatory markers, rather than conventional risk factors, are different between carotid and MCA atherosclerosis. J Neurol Neurosurg Psychiatry 2005;76:1128-1134.

43. Mathur KS, Kashyap SK, Kumar V. Correlation of the extent and severity of atherosclerosis in the coronary and cerebral arteries. Circulation 1963;27:929-934

44. McGarry P, Solberg LA, Guzman MA, Strong JP. Cerebral atherosclerosis in New Orleans. Comparisons of lesions by age, sex, and race. Lab Invest 1985;52:533-539.

45. Santos RD, Nasir K, Rumberger JA, et al. Difference in atherosclerosis burden in different nations and continents assessed by coronary artery calcium. Atherosclerosis 2006;187:378-384.

46. Willey JZ, Rodriguez CJ, Moon YP, et al. Coronary death and myocardial infarction among Hispanics in the Northern Manhattan Study: exploring the Hispanic paradox. Ann Epidemiol 2012; 22:303-309.

47. Arenillas JF, Candell-Riera J, Romero-Farina G, et al. Silent myocardial ischemia in patients with symptomatic intracranial atherosclerosis: associated factors. Stroke 2005;36:1201-1206.

48. Massot A, Pelegri D, Penalba A, Arenillas J, et al. Lipoproteinassociated phospholipase A2 testing usefulness among patients with symptomatic intracranial atherosclerotic disease. Atherosclerosis 2011;218:181-187.

49. Seo W, Yong HS, Koh S, et al. Correlation of coronary artery atherosclerosis with atherosclerosis of the intracranial cerebral artery and the extracranial carotid artery. Eur Neurol 2008;59:292-298.

50. Yoon YE, Chang HJ, Cho I, et al. Incidence of subclinical coronary atherosclerosis in patients with suspected embolic stroke using cardiac computed tomography. Int J Cardiovasc Imaging 2011;27:1035-1044.

51. Hoshino A, Nakamura T, Enomoto S, et al. Prevalence of coronary artery disease in Japanese patients with cerebral infarction impact of metabolic syndrome and intracranial large artery atherosclerosis. Circ J 2008;72:404-408. 